\title{
A Spread Canny Edge Detection Algorithm for Filtering the Historical Inscription Image
}

\author{
P. Selvakumar \\ Research Scholar \\ Department of Computer Science \\ Research and Development Centre, Bharathiar \\ University, Coimbatore
}

\author{
S. Hari Ganesh, PhD \\ Assistant Professor \\ Department of Computer Science \\ HH The Rajah's College (Autonomous), Pudukottai
}

\begin{abstract}
An edge detection of a picture is the basic piece of picture handling, which straightly misrepresented by the picture understanding, picture investigation and acknowledgment. The most imperative administrator for edge detection in picture is the vigilant edge detection administrator. In spite of the fact that there are a few confinements in this administrator. The issues like false edge detection and data of missing edge are come in the Gaussian separating system which is utilized by customary shrewd administrator. The Selection of high and low limits in the conventional watchful administrator are not exact, furthermore it can't bolstered by the self-adaption. To defeat this kind of issue, an advanced calculation is proposed in this paper for the shrewd administrator. In this article, to enhance the separating handling by keeping up the picture edge data, a structure called Spread Canny Edge Detection is proposed.
\end{abstract}

\section{General Terms}

Edge Detection, Digital Image Processing, Filtering.

\section{Keywords}

Canny Edge Detection, Spread Canny Edge Detection, Kmeans, Histogram, Chi-Square Distance, Texture data map.

\section{INTRODUCTION}

The handling of the computerized PC is absolutely done by methods for the Digital Image Processing [1]. The few of PC calculation are utilized on the computerized image for the image handling. For the operations like image morphology, highlight extraction, division, design acknowledgment and so forth on the advanced images, this sort of innovation is generally used. Image preparing is contained by the capable field called Edge location [5] all alone. The image division method which is basically called edge identification strategy, where the image is differentiated into the noteworthy areas or parts. The edges in the images are portrayed by the limits and subsequently an issue of vital critical in the image preparing. In an image, the limit among the two unique areas is happened by edges. Those highlights in an image are seen by the clients in the edge location, when there is the surface speaking to the beginning of another image at the base of one locale or there is a sudden variety in the dim level of an image. The applications like programmed movement controlling frameworks, PC supported surgery conclusion, confront acknowledgment [2], anatomical structure, unique mark acknowledgment, therapeutic imaging, area of items in the satellite image were utilized this procedure basically. For the extraction of the edges, different edge location [3] techniques have been set up. The standard administrators like Prewitt,
Robert, Sobel were right off the bat used for edge location which depends on Gradient work. Since it is likewise amazingly infiltrating to commotion images and the sharp edges from this administrators are unexpectable. The serious mistake of confinement at the bended edges and high probabilityof location of false edges are the two limitations happened in the Marr Hildrith administrator which depends on Laplacian work. To recognize the images that are besmirched with the clamor, Canny Edge discovery calculation is considered as the perfect arrangement which is proposed by John F. Shrewd in the year 1986. This calculation is expected to gives the sharp edges and to lessen the likelihood of recognition of false edge from the image.

\section{LITERATE REVIEW}

The following table 1 depicts the related works on the Edge detection and canny edge detection methods in the field of image processing and its applications.

Table 1. Related works on the Edge Detection methods

\begin{tabular}{|c|c|c|}
\hline $\begin{array}{l}\text { Author } \\
\text { Name }\end{array}$ & Title of the Paper & Methods used \\
\hline $\begin{array}{l}\text { Qian Xu, et } \\
\text { al. [4] }\end{array}$ & $\begin{array}{l}\text { A Distributed Canny } \\
\text { Edge Detector: } \\
\text { Algorithm and FPGA } \\
\text { Implementation }\end{array}$ & $\begin{array}{l}\text { This paper utilizes } \\
\text { the thresholds } \\
\text { based on the local } \\
\text { distribution of the } \\
\text { gradients and } \\
\text { block type }\end{array}$ \\
\hline $\begin{array}{l}\text { Shihu Zhu } \\
\text { [5] }\end{array}$ & $\begin{array}{l}\text { Edge Detection Based } \\
\text { on Multi-structure } \\
\text { Elements Morphology } \\
\text { and Image Fusion }\end{array}$ & $\begin{array}{c}\text { Image Fusion and } \\
\text { Entropy Weighted } \\
\text { Method }\end{array}$ \\
\hline $\begin{array}{l}\text { C.Naga Raju } \\
\text { et al. [6] }\end{array}$ & $\begin{array}{l}\text { Morphological Edge } \\
\text { Detection Algorithm } \\
\text { Based on Multi- } \\
\text { Structure Elements of } \\
\text { Different Directions }\end{array}$ & $\begin{array}{l}\text { Morphology } \\
\text { Gradient } \\
\text { algorithm, }\end{array}$ \\
\hline $\begin{array}{l}\text { Wenshuo } \\
\text { Gao et al. [7] }\end{array}$ & $\begin{array}{l}\text { An Improved Sobel } \\
\text { Edge Detection }\end{array}$ & $\begin{array}{c}\text { Sobel edge } \\
\text { detection operator } \\
\text { and } \\
\text { soft-threshold } \\
\text { wavelet }\end{array}$ \\
\hline $\begin{array}{c}\text { Gentsos, C } \\
\text { et al. [8] }\end{array}$ & $\begin{array}{l}\text { Real-time canny edge } \\
\text { detection parallel } \\
\text { implementation for } \\
\text { FPGAs }\end{array}$ & $\begin{array}{c}\text { Canny Edge } \\
\text { detector with 4- } \\
\text { pixel } \\
\text { parallel } \\
\text { computation } \\
\end{array}$ \\
\hline $\begin{array}{l}\text { Sabina et al. } \\
\text { [9] }\end{array}$ & $\begin{array}{l}\text { A New Edge Detection } \\
\text { Method based on }\end{array}$ & $\begin{array}{l}\text { Image } \\
\text { binarization, }\end{array}$ \\
\hline
\end{tabular}




\begin{tabular}{|c|c|c|}
\hline & $\begin{array}{c}\text { Additions and } \\
\text { Divisions }\end{array}$ & $\begin{array}{c}\text { image } \\
\text { contraction, image } \\
\text { subtraction }\end{array}$ \\
\hline $\begin{array}{c}\text { Tapas } \\
\text { Kumar } \\
{[10]}\end{array}$ & $\begin{array}{c}\text { A Novel Method of } \\
\text { Edge Detection using } \\
\text { Cellular Automata }\end{array}$ & Cellular Automata \\
\hline
\end{tabular}

\section{DEFINITION OF THE PROBLEM}

In the correlation of the human examination and Canny technique, the precision level of edge recognition in Canny is observable however it not upto the level of human investigation. The calculations are incredible at recognizing discernible edges, yet these can happen not simply on the edge of the objects of interest, yet what's more inside them: the articles surface can be caused by the apparent edges. Of these, solitary the past is (generally) critical to us. Envision a situation in which, in the midst of our edge discovery process, we could neglect absolutely of the edges that occur as an element of a surface, leaving only those that are truly question limits.

\section{PROPOSED SPREAD CANNY EDGE DETECTION FRAMEWORK}

The data about the splendor and the surfaces are known as shrewd calculation. In this paper, an endeavor is made to comprehend the splendor and surfaces data issues by the watchful calculation. With the assistance of the data of surface, the edge recognition is ease.

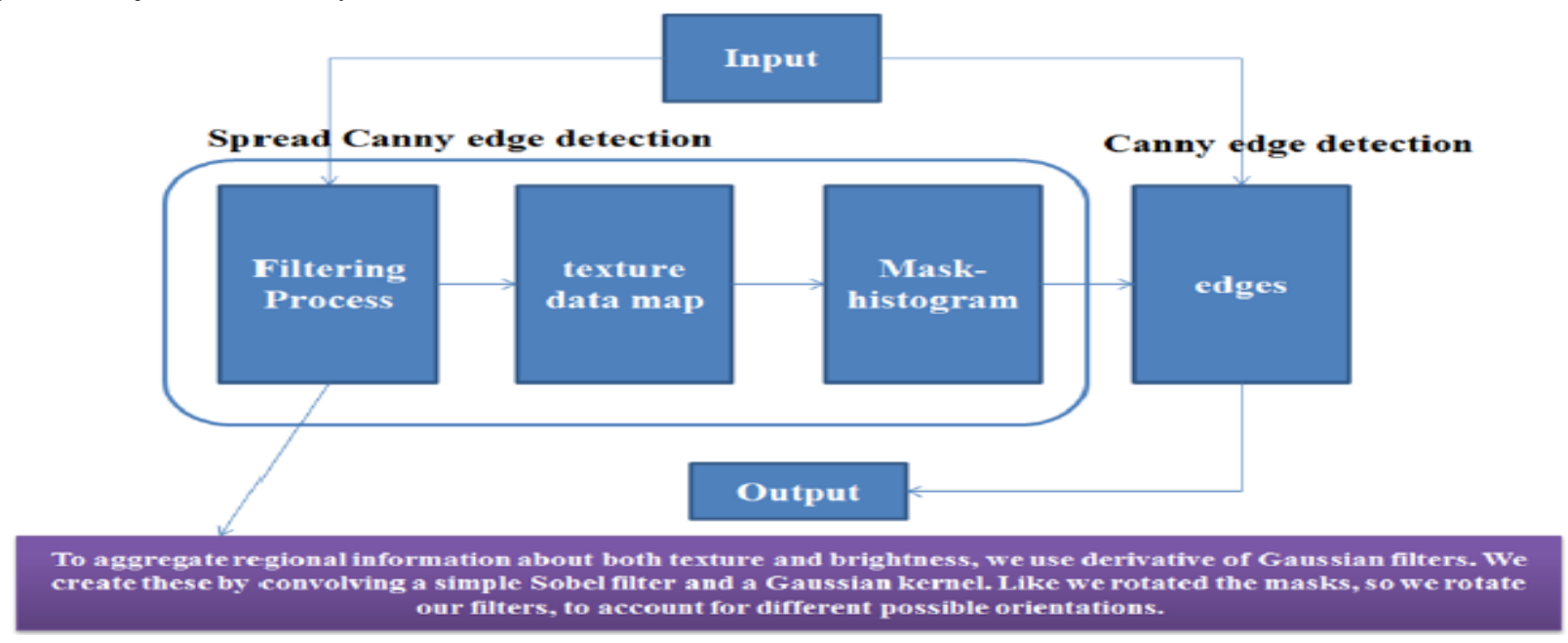

Fig 1: Framework using Spread Canny Edge Detection and Canny Edge Detection

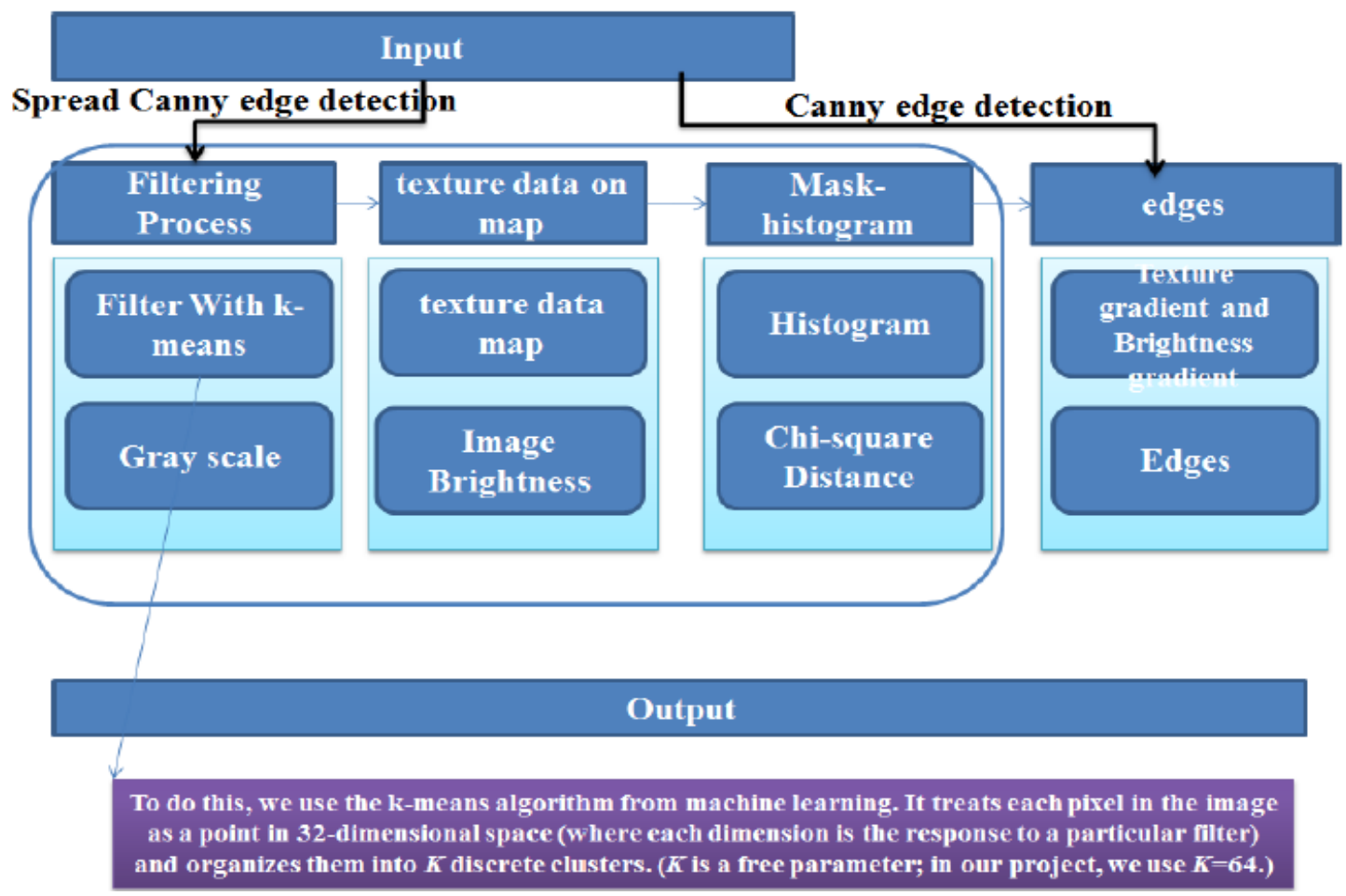

Fig 2: Methods used in Spread Canny Edge Detection and Canny Edge Detection 


\subsection{Filtering Process with K-Means \\ Clustering}

The diverse hugeness edges can't be separated by the major customary techniques for edge recognition and it isn't much perceptible for loud image. However, it works for the images of high caliber. In the first images are dirtied by the commotion which is erratic. For the most part, the accompanying two sorts of commotion are happened in the first images like (I) salt and pepper clamor and (ii) background noise. With respect to the pixels of the image environment, the power or shade of an image are diverse in the salt and pepper commotion. There will be no relationship permitted by the estimation of loud pixel to the encompassing pixels shading. Just a base amount of the image pixels can be aggravated by this sort of commotion. The salt and pepper commotion is inferred by breaking down the images which involves white and dark spots. In the time of 1967, J. MacQueen built up the grouping calculation called K-Means bunching. Relies on the highlights or characteristics, the items can be classified or assembled into the $\mathrm{K}$ (the positive whole number) number of bunches. To limit the whole of squares of separation between the data to the resultant group centroid, the bunching happens. The fundamental purpose behind KMeans bunching is to arrange the information into bunches (gatherings).

Step 1: Start with the value of number of clusters $(k)$

Step 2: Set some primary division that categorizes the data into clusters (k-number of clusters). By systematically or randomly the training samples can be assigned by the following:

- $\quad$ Consider the first training sample (k) as the clusters of single-element.

- By means of the adjacent centroid, the remaining (M-k) samples can be allocated. The gaining cluster centroid can be calculated after every consignment.

Step 3: Consider every sample in order and calculate its distance from every clusters centroid. The cluster which is dropping and attaining the samples can be updated and their centroid can be updated, only when a sample is not presently in the group with the adjoining centroid.

Step 4: Recurrence step 3 until convergence is achieved, that is until a permit over the training sample causes no new tasks.

It is basic to enhance images by changing the force estimations of pixels. Most extreme programming instruments for image preparing have a few inclinations for modifying the nearness of an image by changing over the pixels by means of a lone capacity that plots the estimation of an info dark into the estimation of new yield. It is quiet to spread this with the goal that a handler can determine various diverse image locales and spread an unmistakable plotting capacity to each. Remapping the dim esteems is much of the time called extending on the grounds that it is basic to territory the dim estimations of an image that is excessively dim onto the full arrangement of dark esteems accessible.

\subsection{Mask Histogram Process}

The histogram of a digital image with $\mathrm{D}$ total possible intensity levels in the range $[0, \mathrm{I}]$ is defined as the discrete

function:

$$
\mathrm{H}\left(\mathrm{s}_{\mathrm{j}}\right)=\mathrm{m}_{\mathrm{j}}
$$

Where

$\mathrm{s}_{\mathrm{j}}$ is the intensity level in the interval $[0, \mathrm{I}]$.

$\mathrm{m}_{\mathrm{j}}$ is the number of pixels in the image whose intensity level is $s_{j}$.

$\mathrm{I}:$ is the images.

Histogram Equalization (HE) is a precise standard method for cultivating image contrast [3] [4]. Earlier, this image contrast enrichment technique has been pragmatic to the image of natural scenery and man with worthy outcomes [5]. HE is a procedure that altering the circulation of gray scale value in an image so that it becomes constant. The aim is to gain a uniform spread of the histogram so that each gray scale value has a comparatively identical number of pixels. With a transformation function D [6], Flattening histogram attained by varying the new one (r) gray scale with the (s) gray scale of a pixel. The following equation $r=D$ (s). $S$ can be improved from the inverse transformation function by mathematically as in the equation:

$$
\mathrm{S}=\mathrm{D}^{-1}(\mathrm{r})
$$

where $0<\mathrm{s}<1$. The equation used to calculate the HE can be written as:

$$
\mathrm{K} 0=\operatorname{roun} K_{0}=\operatorname{round}\left(\frac{B k\left(2^{j}-1\right.}{u . h}\right)
$$

$\mathrm{Bk}=$ the cumulative distribution of $\mathrm{i}_{\mathrm{th}}$ grayscale from original image

round $=$ rounding to the nearest

$\mathrm{K}_{0}=$ gray level value from histogram value equalization

$\mathrm{u}=$ width of the image

$1=$ height of the image

This method is also useful for images with both background and foreground are bright or both dark.

\subsection{Edge Detection by using Canny Edge Detection Method}

First of all, we have to clarify what is Edge Detection. Here are some definitions of edge detection: An edge is not a physical entity, just like a shadow. It is where the picture ends and the wall starts. It is where the vertical and the horizontal surfaces of an object meet. It is what happens between a bright window and the darkness of the night. Simply speaking, it has no width. If there were sensor with infinitely small footprints and zero-width point spread functions, an edge would be recorded between pixels within in an image. In reality, what appears to be an edge from the distance may even contain other edges when looked closer. The edge between a forest and a road in an aerial photo may not look like an edge any more in an image taken on the ground. In the ground image, edges may be found around each individual tree. If looked a few inches away from a tree, edges may be found within the texture on the bark of the tree. Edges are scale-dependent and an edge may contain other edges, but at a certain scale, an edge still has no width. Edges characterize boundaries and are therefore a problem of fundamental importance in image processing. Edges in images are areas with strong intensity contrasts - a jump in intensity from one pixel to the next. Edge detecting an image significantly reduces the amount of data and filters out useless information, while preserving the important structural properties in an 
image. The Canny edge detection algorithm is known to many as the optimal edge detector.

\section{RESULT AND DISCUSSION}

In this research paper, Canny Edge Detection and proposed Spread Canny Edge Detection are relatively beneficial for the recovery of significant images from the image databases. From the results indicates that the proposed method offers important concert enhancements in recovery of images. The performance of $\mathrm{k}$-means means in the spread canny edge detection is given as follows: The k-mean clustering affords a qualitatively and minimum error of localization, an intense enhancement in edge detection performance over an existing edge detection method for spotted imagery. The k-mean clustering intended to permit for well-adjusted and fit localized edge strength dimensions in optimistic regions as well as in dark areas. The performance of the k-mean clustering has been demonstrated for edge-detection speckle minimizing anisotropic diffusion.

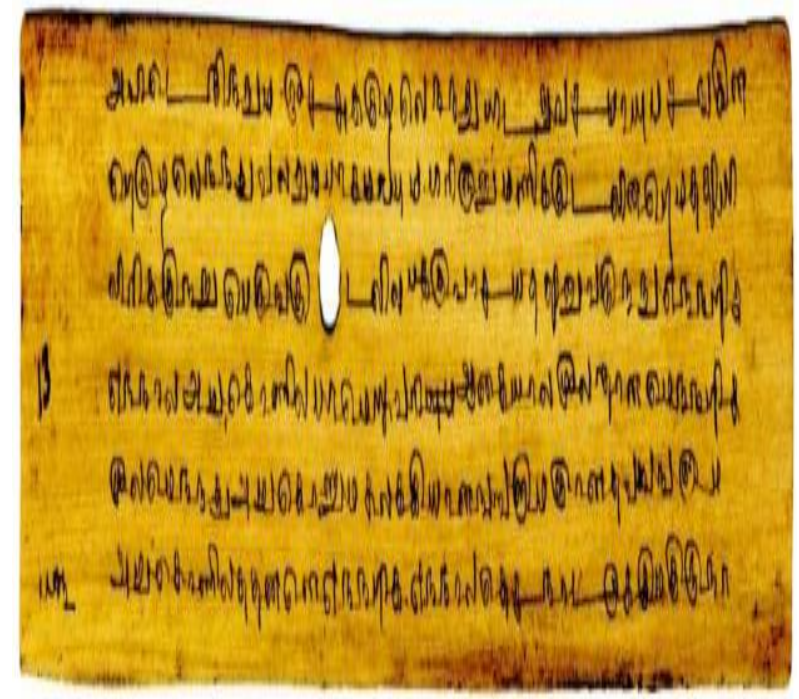

Fig 3: Original Image

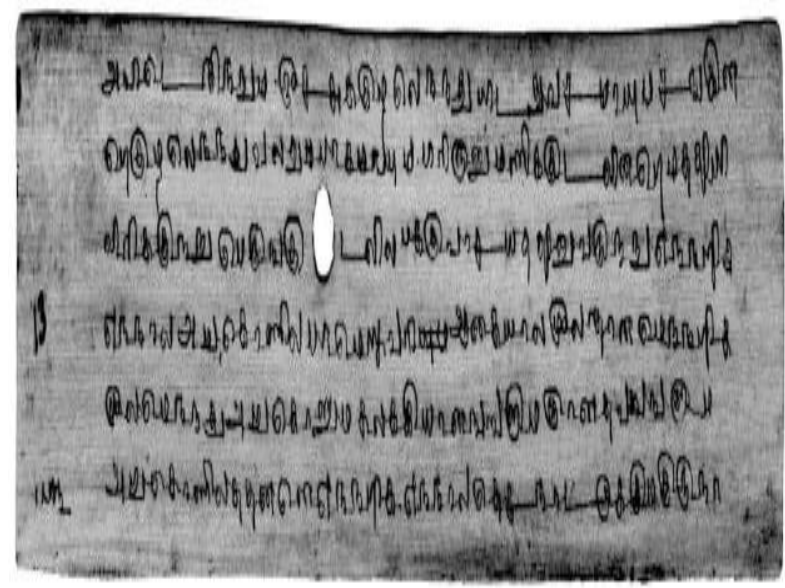

Fig 4: Original image in Gray Scale by using Filtering with k-means

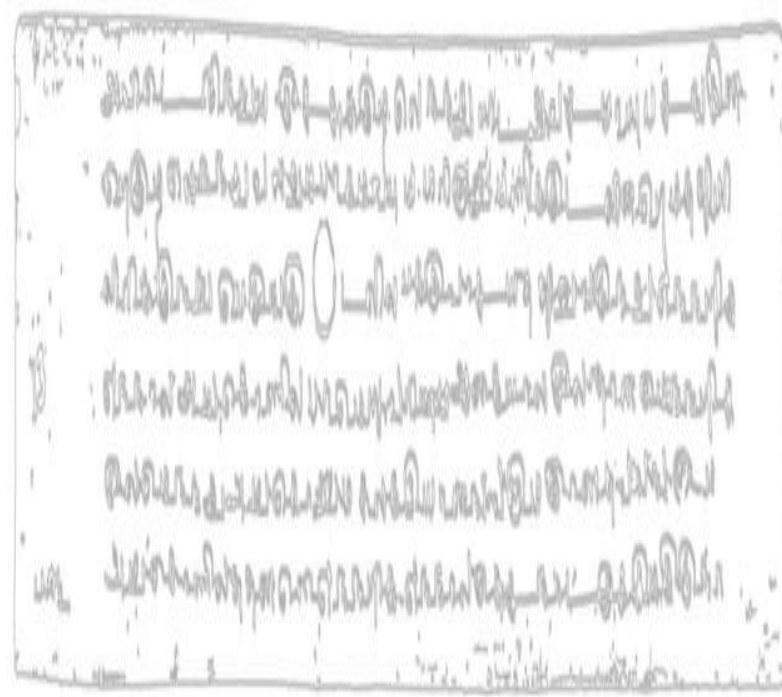

Fig 5: Result obtained from Canny Edge Detection Method

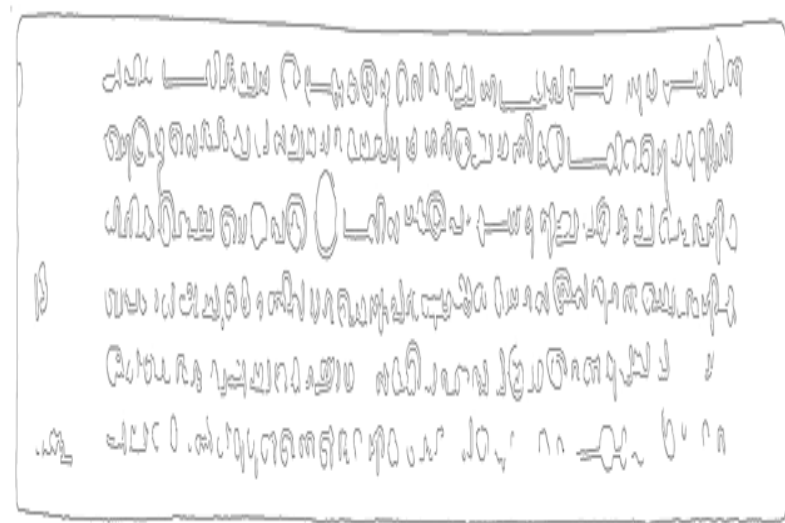

Fig 6: Result obtained by Proposed Spread Canny Edge Detection Method

The following are the advantages of the proposed Spread Canny Edge Detection method

- $\quad$ The Spread Canny algorithm contains a number of adaptable limits, which can disturb the calculation period and efficiency of the algorithm.

- The k-means filter size: the smoothing filter used in the principal stage straightly disturbs the outcomes of the Spread Canny algorithm. Reduced filters cause fewer blurring, and permit recognition of sharp and small lines. A greater filter causes further blurring, spreading out the value of a given pixel over a larger area of the image.

- Thresholds: The important information can be missed when setting the high threshold. Conversely, a threshold set too small will incorrectly recognize inappropriate information (such as noise) as significant. It is tough to provide a common threshold that works well on all images. No irritated and confirmed approach to this difficult up till now.

- This proposed algorithm can be helpful for Complex Computations and time consuming. 


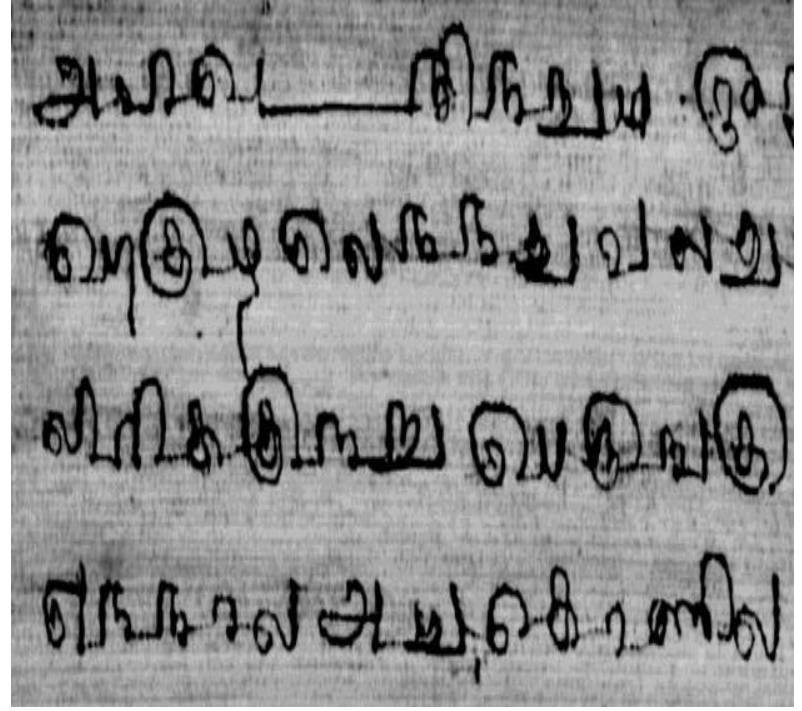

Fig 7: Original Image in Gray Scale with Filtering process using k-means
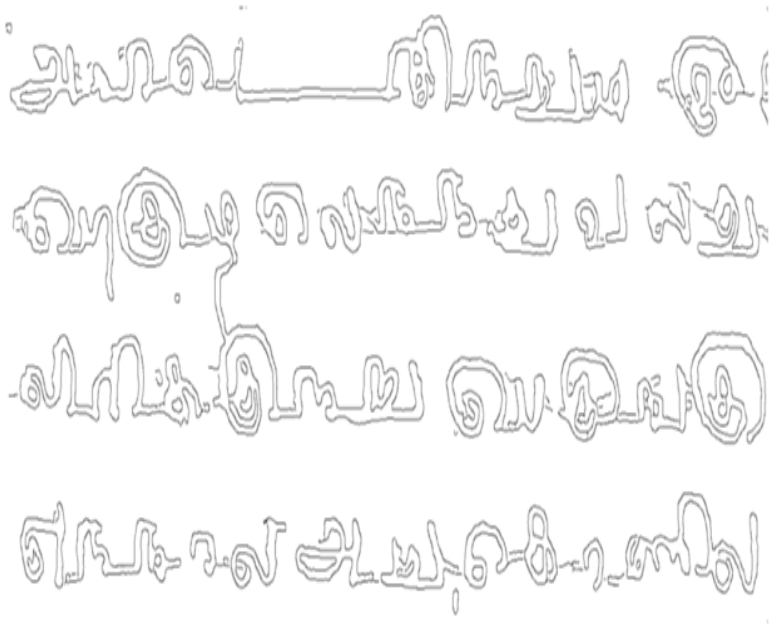

Fig 8: Result obtained from Canny Edge Detection Method
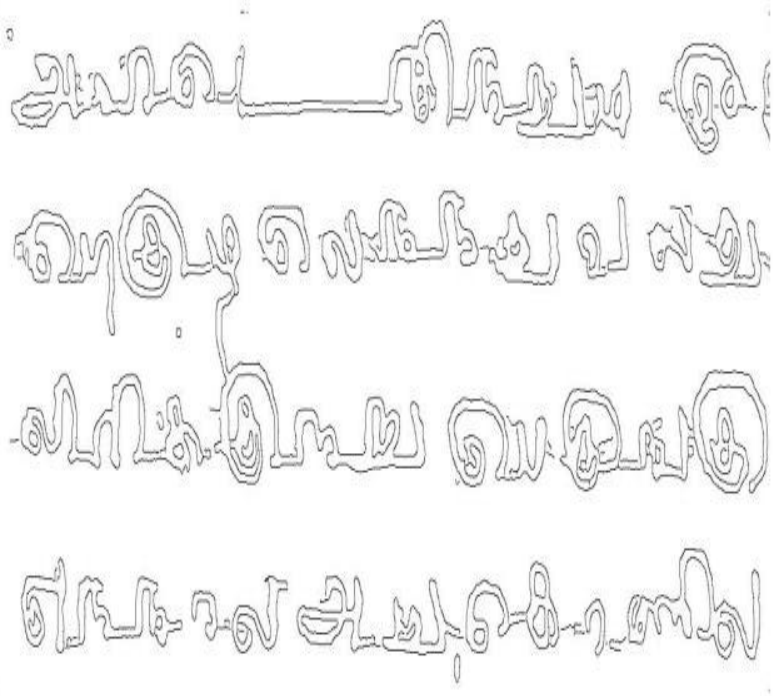

Fig 9: Result obtained from proposed Spread Canny Edge Detection method
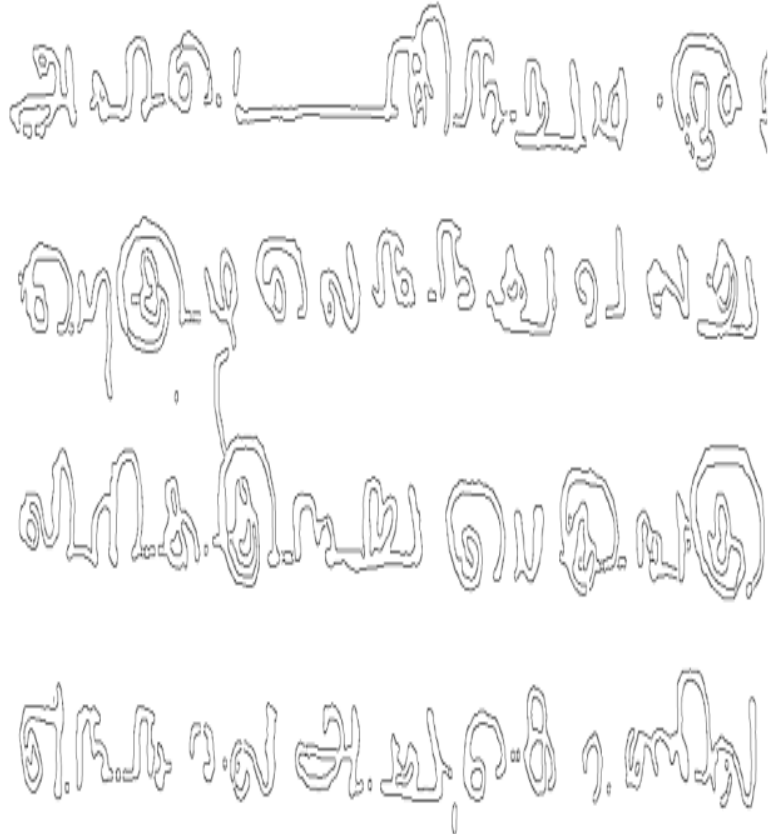

Fig 10: Result obtained by lowering the radius $(2.5)$ in the proposed Spread Canny Edge Detection
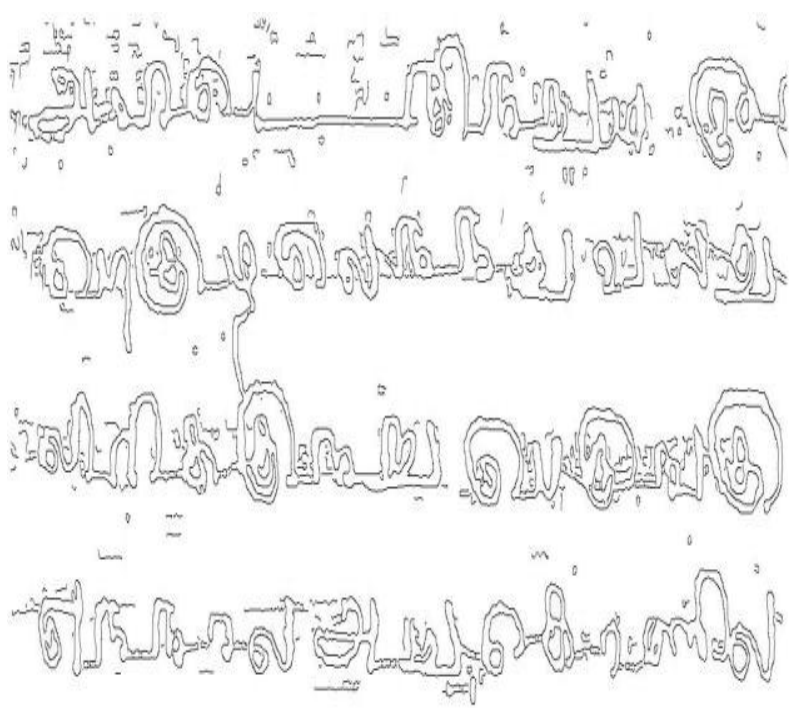

Fig 11: Result obtained by increasing the radius (5) in the proposed Spread Canny Edge Detection

\section{CONCLUSION}

From the above outcomes, the execution of the Spread Canny Edge Detection techniques are superior to the current Canny Edge Detection since it utilizes the K-Means clustering calculation in the separating procedure rather than different channels. Utilization of k-means decreases the clamor, enhances the nature of the image.

\section{REFERENCES}

[1] Mueller, Angelika. "Feature Extraction Image Processing For Computer Vision." Computer 1 (2016): 2.

[2] Mandal, Bappaditya. "Face recognition: Perspectives from the real world." Control, Automation, Robotics and Vision (ICARCV), $201614^{\text {th }}$ International Conference on. IEEE, 2016. 
[3] Hsiao, Yu-Zhe, and Soo-Chang Pei. "Edge detection, color quantization, segmentation, texture removal, and noise reduction of color image using quaternion iterative filtering." J. Electronic Imaging 23.4 (2014): 043001.

[4] Qian Xu, Srenivas Varadarajan, Chaitali Chakrabarti, Fellow, IEEE, and Lina Karam, Fellow, IEEE "A Distributed Canny Edge Detector: Algorithm and FPGA Implementation" IEEE Transactions on Image processing, Vol. 23, No. 7, 2014.

[5] Shihu Zhu, "Edge Detection Based on Multi-structure Elements Morphology and Image Fusion", IEEE, 978-14244-9600-vol-6, 2011.

[6] C.Naga Raju, S.Naga Mani, G.rakesh Prasad, S.Sunitha, "Morphological Edge Detection Algorithm Based on Multi-Structure Elements of Different Directions", International Journal of Information and Communication Technology Research Volume 1 No. 1, May 2011.
[7] Wenshuo Gao, Lei Yang, Xiaoguang Zhang, Huizhong Liu, “An Improved Sobel Edge Detection", IEEE, 978-14244-5540-vol-9,2010.

[8] Gentsos, C. Sotiropoulou, S. Nikolaidis, and N. Vassiliadis, "Real-time canny edge detection parallel implementation for FPGAs," in Proc. IEEE ICECS, Dec. 2010, pp. 499-502

[9] Sabina Priyadarshini, Gadadhar Sahoo, "A New Edge Detection Method based on Additions and Divisions", International Journal of Computer Applications (0975 8887) Volume 9- No.10, November 2010.

[10] Tapas Kumar, G. Sahoo, "A Novel Method of Edge Detection using Cellular Automata", International Journal of Computer Applications (0975 - 8887) Volume 9- No.4, November 2010. 